\title{
Plasma miR-22-5p, miR-132-5p, and miR-150-3p Are Associated with Acute Myocardial Infarction
}

\author{
Huixian Li $(\mathbb{D}$, Pengxiang Zhang, Fangjiang Li $(\mathbb{D}$, Guili Yuan, \\ Xiaoyuan Wang, Aiai Zhang, and Feixing Li \\ Department of Cardiology, The First Affiliated Hospital of Hebei North University, Zhangjiakou City, Hebei Province 075000, China \\ Correspondence should be addressed to Fangjiang Li; lfj6789@163.com
}

Received 23 December 2018; Accepted 31 March 2019; Published 24 April 2019

Academic Editor: Mouldy Sioud

Copyright (c) 2019 Huixian Li et al. This is an open access article distributed under the Creative Commons Attribution License, which permits unrestricted use, distribution, and reproduction in any medium, provided the original work is properly cited.

\begin{abstract}
Circulating microRNAs (miRNAs) are potential biomarkers for cardiovascular diseases. Our study aimed to determine whether miR-22-5p, miR-132-5p, and miR-150-3p represent novel biomarkers for acute myocardial infarction (AMI). Plasma samples were isolated from 35 AMI patients and 55 matched controls. Total RNA was extracted, and quantitative real-time PCR and ELISA were performed to investigate the expressions of miRNAs and cardiac troponin I (cTnI), respectively. We found that plasma levels of miR-22-5p and miR-150-3p were significantly higher during the early stage of AMI and their expression levels peaked earlier than cTnI. Conversely, circulating miR-132-5p was sustained at a low level during the early phase of AMI. All three circulating miRNAs were correlated with plasma cTnI levels. A receiver operating characteristic (ROC) analysis suggested that each single miRNA had considerable diagnostic efficacy for AMI. Moreover, combining the three miRNAs improved their diagnostic efficacy. Furthermore, neither heparin nor medications for coronary heart disease (CHD) affected plasma levels of miR-22-5p and miR-132$5 p$, but circulating miR-150-3p was downregulated by medications for CHD. We concluded that plasma miR-22-5p, miR-132-5p, and miR-150-3p may serve as candidate diagnostic biomarkers for early diagnosis of AMI. Moreover, a panel consisting of these three miRNAs may achieve a higher diagnostic value.
\end{abstract}

\section{Introduction}

Acute myocardial infarction (AMI) is one of the leading causes of death worldwide [1]. Diagnosing AMI early and accurately facilitates the immediate initiation of reperfusion therapy, which potentially reduces its mortality rate. Some biomarkers, such as creatine kinase-MB (CK-MB) and cardiac troponins ( $\mathrm{cTnI} / \mathrm{cTnT}$ ), have been widely reported to be gold-standard indicators for the clinical diagnosis of AMI [2]. However, novel biomarkers, including molecular and genetic biomarkers, are being investigated for their ability to improve diagnostic accuracy and provide more prognostic information relevant to AMI [3].

MicroRNAs (miRNAs or miRs) are endogenous, small (19-25 nucleotides) single-stranded, noncoding RNAs that play a critical role in regulating gene expression by binding to the $3^{\prime}$ untranslated regions ( $3^{\prime}$-UTR) of target gene messenger RNAs (mRNAs) [4-6]. Numerous miRNAs have been demonstrated to play important roles in cell biological and pathological processes, including proliferation, differentiation, and apoptosis [7]. Notably, miRNAs are stable in various biological fluids, including serum, plasma, and urine. Reports have suggested that it may actually be possible to stabilize miRNAs under extreme conditions, including boiling, high or low $\mathrm{pH}$, or extended storage and freezethaw cycles, by inducing the formation of protein-miRNA or chemical-miRNA complexes [8]. An increasing amount of evidence has shown that circulating miRNAs (e.g., miR1, miR-133a, miR-499, and miR-208b) have the potential to serve as alternative biomarkers for the diagnosis of AMI $[9,10]$.

As a cardiac- and skeletal muscle-enriched miRNA, miR-22 has been reported to participate in cardiomyocyte hypertrophy and cardiac remodeling in response to stress [11]. The miR-132/212 cluster has been confirmed to play a critical role in cardiac hypertrophy [12]. As a dominant member of the miR-132/212 family, miR-132 negatively regulates cardiac function during cardiac remodeling by inducing 


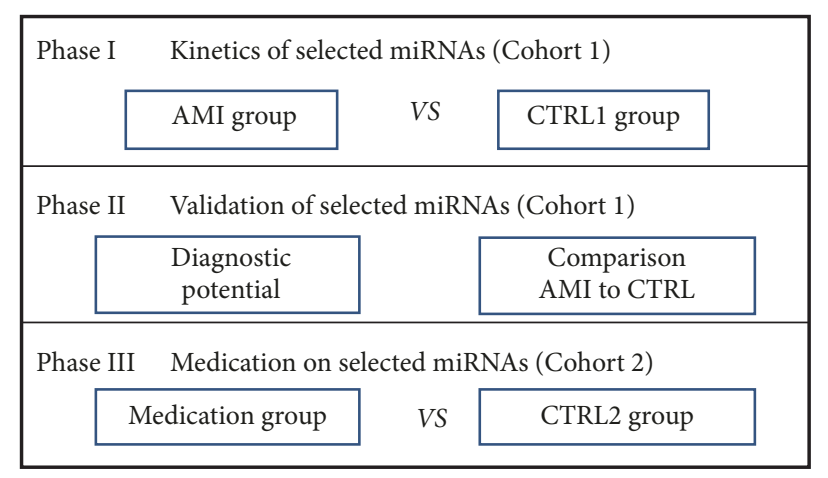

Figure 1: A flow chart of the study design strategy. The present study was conducted in the three following phases: (i) initial determination phase: we evaluated the dynamic expressions of the selected miRNAs in plasma obtained from patients with acute myocardial infarction (AMI) and control subjects (CTRL1), (ii) validation phase: we evaluated the potential diagnostic value of the selected miRNAs for discriminating AMI patients from controls, and (iii) determination phase: we evaluated the effects of heparin and medications on the plasma levels of selected miRNAs. CRTL1, control subjects presented with chest pain without coronary artery diseases (CHD); CRTL2, paroxysmal supraventricular tachycardia (PSVT) patients without medications for CHD; Medication, patients taking medications for CHD.

angiogenesis. miR-150, a miRNA that is closely associated with inflammation, has been implicated in the pathogenesis of various cardiovascular diseases [13-15]. miR-150 has been shown to regulate high glucose-induced cardiomyocyte hypertrophy by targeting the transcriptional coactivator p300 [16]. Overall, these studies indicate that all three miRNAs play important roles in various cardiovascular diseases. Interestingly, a recent microarray analysis showed that the expression levels of miR-22, miR-132, and miR-150 were obviously dysregulated in patients with AMI [10]. However, the clinical significance of circulating miR-22-5p, miR-132$5 \mathrm{p}$, and miR-150-3p in patients with AMI remains unclear. In this study, we investigated the dynamic expressions of miR22-5p, miR-132-5p, and miR-150-3p in plasma obtained from patients in the early phase of AMI and explored the potential diagnostic value of these markers. Our findings suggest that circulating miR-22-5p, miR-132-5p, and miR-150-3p may be promising biomarkers for the early stage of AMI without affected by the affected by heparin or cardiovascular drugs. Furthermore, a composite of these miRNAs may provide superior predictive value.

\section{Results}

2.1. Patient Characteristics. Two cohorts comprising 180 participants were enrolled in the present study (Figure 1). In the first cohort, we enrolled and assessed the clinical characteristics of 35 AMI patients and 55 control subjects. These results are listed in Table 1 . There were no significant differences between the control subjects and the AMI patients for most of the considered clinical variables, including total cholesterol (TC), total triglycerides (TG), highdensity lipoprotein (HDL), low-density lipoprotein (LDL), systolic/diastolic blood pressure (SBP/DBP), body mass index (BMI), creatinine ( $\mathrm{Cr})$, history of diabetes, and smoking status. However, white blood cell counts and CK-MB were significantly higher in the AMI patients than in the control subjects, which is in agreement with previous studies. Additionally, the medications that were used to treat AMI were significantly different between the AMI patients and control subjects.

Subsequently, we explored the potential effects of medications for coronary heart disease (CHD) on the expressions of selected miRNAs in the second cohort (40 patients with CHD using medication and 50 patients with paroxysmal supraventricular tachycardia). There were significant differences between the two groups in the following characteristics: history of hypertension or hyperlipidemia and related medications (Table 2).

2.2. Expression Patterns and the Correlation of Plasma miRNAs and cTnI. To investigate the expression patterns of selected miRNAs (miR-22-5p, miR-132-5p, and miR-150-3p) and $c$ TnI during the early phase of AMI, we used quantitative real-time PCR (qRT-PCR) and enzyme-linked immunosorbent assay (ELISA) to measure the expression levels of these miRNAs and CTnI in plasma obtained from AMI patients and control subjects. Overall, the expression levels of circulating miR-22-5p and miR-150-3p were significantly higher in the AMI patients than in the control subjects, whereas miR-132$5 \mathrm{p}$ presented the opposite trend (Table 3 ). Compared to the controls, the expression of plasma miR-22-5p in the AMI patients increased and reached the peak at T0 (36.47 fold) and then gradually decreased but still upregulated till $72 \mathrm{~h}$ after T0 (Figure 2(a)). Meanwhile, plasma miR-150-3p also upregulated and achieved peak at T0 (4.09 fold), but the expression level quickly decreased and return to normal at $48 \mathrm{~h}$ after T0 (Figure 2(c)). In contrast, circulating miR-132$5 \mathrm{p}$ in plasma exhibited a sustained low level of expression at all time-points in the early stage of AMI of AMI patients compared to the control subjects (Figure 2(b)). Plasma cTnI levels were also measured in the same samples, which showed that the expression levels of cTnI were higher in the AMI patients at T0, achieved peak at $12 \mathrm{~h}$ (2518 fold), and then gradually decreased but still upregulated till $72 \mathrm{~h}$ compared to the control subjects (Figure 2(d)) (Table 3).

Firstly, we found that the relative expression of cTnI reached its peak at $12 \mathrm{~h}$ after T0, which was later than the 
TABLE 1: Clinical characteristics of the AMI patients and control subjects in the first cohort.

\begin{tabular}{|c|c|c|c|}
\hline Variable & $\begin{array}{l}\text { AMI group } \\
(\mathrm{n}=35)\end{array}$ & $\begin{array}{l}\text { Control group } \\
(\mathrm{n}=55)\end{array}$ & $p$-value \\
\hline Male/Female (n/n) & $15 / 20$ & $27 / 28$ & 0.26 \\
\hline Age (years) & $60.86 \pm 11.25$ & $56.36 \pm 12.36$ & 0.09 \\
\hline Body mass index (kg/m2) & $25.43 \pm 2.82$ & $24.52 \pm 2.52$ & 0.12 \\
\hline \multicolumn{4}{|l|}{ Smoking status } \\
\hline Current smoker (\%) & $6(17 \%)$ & $4(7 \%)$ & 0.15 \\
\hline Former smoker (\%) & $12(34 \%)$ & $11(20 \%)$ & 0.13 \\
\hline Hypertension, n (\%) & $11(31 \%)$ & $12(22 \%)$ & 0.31 \\
\hline Hyperlipidaemia, n (\%) & $13(37 \%)$ & $14(25 \%)$ & 0.24 \\
\hline Diabetes mellitus, n (\%) & $5(14 \%)$ & $6(11 \%)$ & 0.63 \\
\hline $\mathrm{WBC}\left(\mathrm{x} 10^{9} / \mathrm{L}\right)$ & $9.35 \pm 1.05$ & $5.84 \pm 1.28$ & $<0.01 * *$ \\
\hline SBP (mmHg) & $129.01 \pm 15.32$ & $128.52 \pm 10.69$ & 0.09 \\
\hline DBP $(\mathrm{mmHg})$ & $74.21 \pm 6.59$ & $76.90 \pm 7.87$ & 0.10 \\
\hline Heart rate (beats/minutes) & $75.42 \pm 12.57$ & $74.05 \pm 12.05$ & 0.61 \\
\hline $\mathrm{TC}(\mathrm{mmol} / \mathrm{L})$ & $4.84 \pm 0.73$ & $4.46 \pm 1.09$ & 0.07 \\
\hline $\mathrm{HDL}(\mathrm{mmol} / \mathrm{L})$ & $1.37 \pm 0.17$ & $1.39 \pm 0.19$ & 0.61 \\
\hline $\mathrm{LDL}(\mathrm{mmol} / \mathrm{L})$ & $3.13 \pm 0.55$ & $2.93 \pm 0.68$ & 0.15 \\
\hline TG (mmol/L) & $1.64 \pm 0.69$ & $1.71 \pm 0.75$ & 0.66 \\
\hline $\mathrm{Cr}(\mathrm{umol} / \mathrm{L})$ & $74.27 \pm 15.02$ & $71.28 \pm 10.96$ & 0.28 \\
\hline CK-MB (IU/L) & $195.67 \pm 63.20$ & $8.56 \pm 1.87$ & $<0.01 * *$ \\
\hline \multicolumn{4}{|l|}{ Medications } \\
\hline ACE inhibitors (\%) & $22(63 \%)$ & $8(15 \%)$ & $<0.01 * *$ \\
\hline Beta-blockers (\%) & $27(77 \%)$ & $6(11 \%)$ & $<0.01 * *$ \\
\hline Nitrates (\%) & $25(71 \%)$ & 1 & $<0.01 * *$ \\
\hline Statins (\%) & $35(100 \%)$ & $14(25 \%)$ & $<0.01 * *$ \\
\hline Aspirin (\%) & $35(100 \%)$ & $8(15 \%)$ & $<0.01 * *$ \\
\hline Clopidogrel (\%) & $35(100 \%)$ & I & $<0.01 * *$ \\
\hline
\end{tabular}

Data are shown as the mean $\pm \mathrm{SD}, * p<0.05, * * p<0.01$ versus control subjects.

peaks for miR-22-5p and miR-132-5p (Figures 3(a)-3(c)). Meanwhile, the result showed that circulating miR-22-5p ( $\mathrm{r}$ $=0.48, \mathrm{p}<0.01)$ and $\mathrm{miR}-150-3 \mathrm{p}(\mathrm{r}=0.46, \mathrm{p}<0.01)$ levels were both positively correlated with cTnI levels. In contrast, plasma miR-132-5p and cTnI were negatively correlated $(\mathrm{r}=$ $-0.62, \mathrm{p}<0.01$ ) (Figures 3(d)-3(f)).

2.3. Diagnostic Efficiency of miRNAs in the Early Stage of AMI. To conveniently evaluate the power of the selected miRNAs to predict AMI, we converted the expression levels of these miRNAs into single score or combined score and then performed a receiver operating characteristic (ROC) analysis, as previously described $[17,18]$.

The mean scores for miR-22-5p were 3.13 (T0), 2.99 (4h), 2.88 ( $8 \mathrm{~h}), 2.94$ (12h), 2.93 (24h), 2.88 (48h), and $2.70(72 \mathrm{~h})$ in the AMI group and $1.49,1.48,1.45,1.51,1.61$, 1.47 , and 1.54, respectively, in the control group (Figures $4(\mathrm{a})-4(\mathrm{~g}))$. The mean scores for miR-132-5p were 2.78 (T0), 3.18 ( $4 \mathrm{~h}), 2.92$ ( $8 \mathrm{~h}), 3.08$ (12 h), 2.85 ( $24 \mathrm{~h}), 2.96$ (48 h), and $2.71(72 \mathrm{~h})$ in the AMI group and 1.34, 1.50, 1.35, 1.38, 1.48, 1.43 , and 1.44, respectively, in the control group (Figures $5(\mathrm{a})-5(\mathrm{~g}))$. The mean scores for miR-150-3p were 4.15 (T0), $3.75(4 \mathrm{~h}), 3.95(8 \mathrm{~h}), 3.71(12 \mathrm{~h})$, and $3.58(24 \mathrm{~h})$ in the AMI group and $1.86,1.80,1.91,1.92$, and 1.84 , respectively, in the control group (Figures 6(a)-6(e)). However, when the three circulating miRNAs were combined, the mean scores for the combined miRNAs were 3.24 (T0), 3.19 (4h), 3.17 $(8 \mathrm{~h}), 3.20(12 \mathrm{~h})$, and $3.01(24 \mathrm{~h})$ in the AMI group and 1.72, $1.74,1.71,1.74$, and 1.79 , respectively, in the control group (Figures 7(a)-7(e)).

The ROC curve analysis indicated that miR-22-5p, miR132-5p, and miR-150-3p (Figures 4(h)-4(n), 5(h)-5(n), and 6(f) $-6(j)$ ) separately showed a moderate power (sensitivity and specificity) for distinguishing value for the AMI during the early phase of chest pain. However, when these three miRNA were combined, A panel of these miRNAs presented higher AUC values than the single one (Figures $7(\mathrm{f})-7(\mathrm{j})$ ) (Table 4).

2.4. The Impact of Heparin and Medications on miRNAs Expression. It has been reported that heparin and medications might affect the expression of some circulating miRNAs, potentially limiting the diagnostic reliability of affected miRNAs [19]. Hence, we firstly investigated whether heparin impacted the levels of the selected miRNAs during the PCI procedure in AMI patients. The results showed that there were no significant differences between pre-PCI and post-PCI plasma samples in the expression levels of the 
TABLE 2: Clinical characteristics of the enrolled subjects in the second cohort.

\begin{tabular}{|c|c|c|c|}
\hline Variable & $\begin{array}{l}\text { Medication group } \\
(\mathrm{n}=40)\end{array}$ & $\begin{array}{l}\text { Control group } \\
(\mathrm{n}=50)\end{array}$ & $p$-value \\
\hline Male/Female (n/n) & $17 / 23$ & $20 / 30$ & 0.24 \\
\hline Age (years) & $50.86 \pm 12.30$ & $46.36 \pm 10.25$ & 0.06 \\
\hline Body mass index $\left(\mathrm{kg} / \mathrm{m}^{2}\right)$ & $24.31 \pm 2.34$ & $23.74 \pm 1.95$ & 0.21 \\
\hline \multicolumn{4}{|l|}{ Smoking status } \\
\hline Current smoker (\%) & $6(15 \%)$ & $4(8 \%)$ & 0.33 \\
\hline Former smoker (\%) & $7(18 \%)$ & $4(8 \%)$ & 0.17 \\
\hline Hypertension history, n (\%) & $17(43 \%)$ & $8(16 \%)$ & $<0.01 * *$ \\
\hline Hyperlipidaemia history, n (\%) & $25(63 \%)$ & $7(14 \%)$ & $<0.01 * *$ \\
\hline Diabetes mellitus, n (\%) & $4(10 \%)$ & $3(6 \%)$ & 0.70 \\
\hline $\mathrm{WBC}\left(\mathrm{x} 10^{9} / \mathrm{L}\right)$ & $5.75 \pm 1.15$ & $5.64 \pm 1.24$ & 0.67 \\
\hline Heart rate (beats/minutes) & $72.42 \pm 10.86$ & $75.05 \pm 9.62$ & 0.23 \\
\hline $\mathrm{Cr}(\mathrm{umol} / \mathrm{L})$ & $73.25 \pm 14.38$ & $69.72 \pm 13.40$ & 0.23 \\
\hline \multicolumn{4}{|l|}{ Medications } \\
\hline ACE inhibitors (\%) & $14(35 \%)$ & $5(10 \%)$ & $<0.01 * *$ \\
\hline Beta-blockers (\%) & $11(28 \%)$ & $3(6 \%)$ & $<0.01 * *$ \\
\hline Nitrates $(\%)$ & $5(13 \%)$ & I & $<0.01 * *$ \\
\hline Statins (\%) & $25(63 \%)$ & $7(14 \%)$ & $<0.01 * *$ \\
\hline Aspirin (\%) & $10(25 \%)$ & $3(6 \%)$ & $0.02 *$ \\
\hline Clopidogrel (\%) & $6(15 \%)$ & I & $<0.01 * *$ \\
\hline
\end{tabular}

Data are shown as the mean $\pm \mathrm{SD}, * p<0.05, * * p<0.01$ patients for medications versus control subjects.

TABLE 3: The relative expression of plasma miRNAs and cTnI in early stage of AMI.

\begin{tabular}{|c|c|c|c|c|c|c|c|}
\hline Fold change & AMI (0h) & AMI (4h) & AMI (8h) & AMI (12h) & AMI (24h) & AMI (48h) & AMI (72h) \\
\hline miR-22-5p & $36.47 \pm 9.11 *$ & $31.81 \pm 6.89 *$ & $19.37 \pm 5.09 *$ & $22.38 \pm 5.89 *$ & $11.71 \pm 4.09 *$ & $14.06 \pm 5.75 *$ & $8.72 \pm 2.52 *$ \\
\hline miR-132-5p & $0.47 \pm 0.08 *$ & $0.36 \pm 0.06 *$ & $0.39 \pm 0.12 *$ & $0.29 \pm 0.13 *$ & $0.52 \pm 0.09 *$ & $0.42 \pm 0.09 *$ & $0.56 \pm 0.06 *$ \\
\hline miR-150-3p & $4.09 \pm 1.20 *$ & $2.93 \pm 0.64 *$ & $3.48 \pm 0.80 *$ & $2.19 \pm 0.47 *$ & $1.84 \pm 0.44 *$ & $1.60 \pm 0.32$ & $1.57 \pm 0.25$ \\
\hline cTnI & $1578 \pm 769 *$ & $2001 \pm 940 *$ & $2198 \pm 1015 *$ & $2518 \pm 1200 *$ & $1597 \pm 767 *$ & $1050 \pm 518 *$ & $654 \pm 291 *$ \\
\hline
\end{tabular}

Data are shown as the mean $\pm \mathrm{SD}, * p<0.05$, plasma expression of miR-22-5p, miR-132-5p, miR-150-3p, and cTnI at different time points in early stage of AMI versus control subjects.

three miRNAs, suggesting that heparin did not impact the expression of these selected miRNAs (Figure 8).

In the initial phase of the present study, we observed that medications (e.g., angiotensin converting enzyme inhibitors (ACEI), beta-blockers, nitrates, statins, aspirin, or clopidogrel) used to treat AMI were significantly different between the patients with AMI and the control subjects. Consequently, to determine whether these medications affect plasma miRNAs expression, we enrolled an additional well-matched cohort in which we analyzed the expression levels of miR22-5p, miR-132-5p, and miR-150-3p. However, only circulating miR-150-3p was significantly different between the two groups, indicating that these medications may reduce the expression of plasma miR-150-3p and thereby be partially involved in its early regression to baseline levels (Figure 9).

\section{Discussion}

In the present study, we provided evidence that circulating miR-22-5p, miR-132-5p, and miR-150-3p might serve as novel promising diagnostic biomarkers for the early diagnosis of
AMI. We showed that plasma miR-22-5p and miR-150-3p levels were markedly elevated in the early phase of AMI and that they reached peak expression earlier than cTnI, whereas the expression of miR-132-5p displayed a sustained downward trend during the early phase of AMI. Furthermore, we demonstrated that miR-22-5p, miR-132-5p, and miR-150-3p each individually showed a moderate ability to discriminate AMI patients from controls, while a multimiRNA approach presented higher discriminatory power than any single miRNA. Additionally, we proved that the expressions of these miRNAs were not influenced by heparin, whereas medications for CHD may reduce the expression of plasma miR-150-3p during the early stage of AMI. There is no doubt that cTnI has been widely used as indicators for the clinical early diagnosis of AMI, however which may be unable to reliably rule in or rule out AMI immediately on admission for early stage of patients with chest pain. Our investigation showed that selected circulating miRNA had an earlier upregulation and higher diagnostic accuracy for AMI, and a combined miRNAs might sever as excellent potential early diagnostic biomarker for AMI in future. 


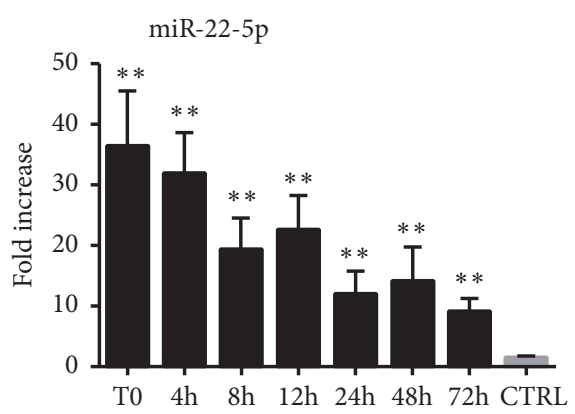

(a)

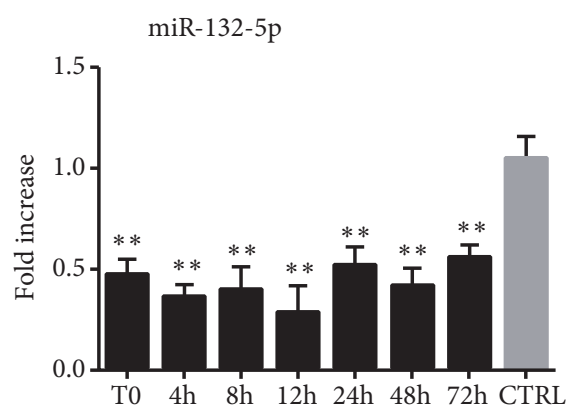

(b)

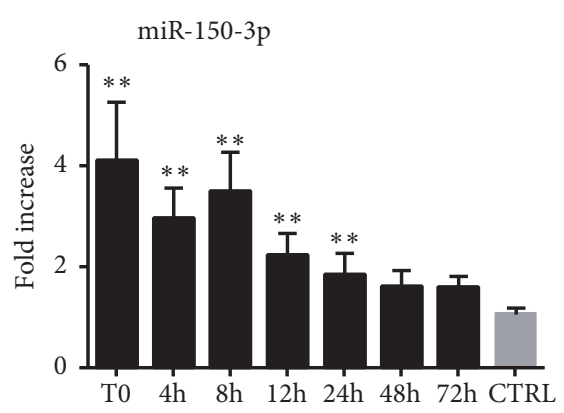

(c)

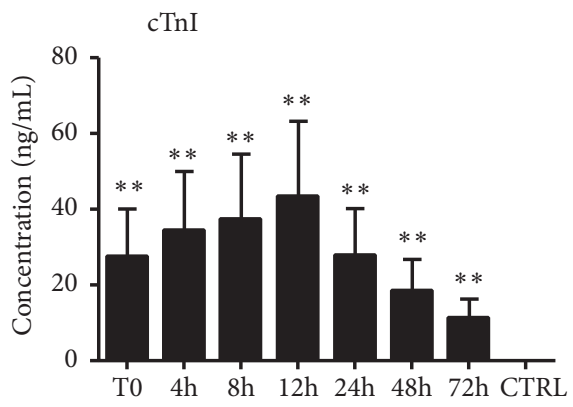

(d)

Figure 2: The plasma levels of miRNAs (quantitative real-time PCR) and cTnI (immunoassay) in the AMI and control groups. The plasma levels of miR-22-5p (a), miR-132-5p (b), miR-150-3p (c), and cTnI (d) in the AMI and control groups. AMI, patients with acute myocardial infarction; CRTL, control subjects; T0, the initial blood sample collection time after the onset of AMI symptoms. Data are shown as the mean $\pm \mathrm{SD}, * \mathrm{p}<0.05, * * \mathrm{p}<0.01$ versus CTRL. AMI, patients with acute myocardial infarction, $\mathrm{n}=35 ; \mathrm{CRTL}$, control subjects, $\mathrm{n}=55 ;$ cardiac troponin I (cTnI).

A large number of studies have shown that miRNAs are implicated in the pathogenesis of various cardiovascular diseases or disorders [20-22]. Moreover, accumulating evidence has indicated that circulating miRNAs, including heart-, vascular-, and endothelium-enriched miRNAs, might serve as potential biomarkers for various cardiovascular diseases, including AMI, unstable angina pectoris (UAP), atrial fibrillation acute myocarditis, heart failure, and acute pulmonary embolism [19, 23-27]. So far, most published studies investigating the use of miRNAs as biomarkers for AMI have focused mainly on myocardium-derived miRNAs $[3,10]$. However, a variety of physiological and pathological changes occur during the AMI process, including disordered endothelial function, plaque erosion/rupture, platelet aggregation, oxidative stress, inflammatory reactions, and myocardial cell injury/necrosis $[18,28]$. A recent study by Wang et al. [18] showed that miRNAs that originate from various cell types during the early course of AMI might sever as novel biomarkers for AMI.

It was previously reported that all of the miRNAs selected for this study are involved in the functional regulation of cardiovascular-derived cells and cardiovascular diseases. As a cardiac-abundant miRNA, miR-22 is critically involved in stress-induced cardiac hypertrophy and remodeling [11]. It has been reported to induce cardiac hypertrophy, in part by targeting PTEN on cardiomyocytes [29]. Yang J. et al. found that miR-22 also protects cardiomyocytes from apoptosis by decreasing the expression of $\mathrm{CBP} / \mathrm{p} 300$ and acetylated p53 [30]. Additionally, circulating miR-22 has been used as a potential predictive marker for several diseases, including chronic nervous lesions, osteosarcoma and aortic stenosis $[31,32]$. As an endothelial cell-specific miRNA, miR-132 plays crucial roles in the regulation of angiogenesis-stimulating factors and the angiogenic process by directly targeting Rasal, Spred1, and p120RasGAP [12, 33, 34]. Eskildsen T. V. et al. found that miR-132 plays a critical role in regulating cardiovascular functions, such as cardiac hypertrophy, heart failure and blood pressure, by targeting AT1R [35]. miR-150, an inflammation-associated miRNA, was also reported to be involved in AMI-induced injury by regulating monocyte migration and the production of proinflammatory cytokines [36]. Additionally, circulating miR-150 was reported to be associated with left ventricular contractility post-MI [37]. Moreover, previous studies have shown that circulating miR150 may be a novel biomarker for platelet activation and disorders in patients with UAP [38]. Interestingly, in the present study, we found that plasma miR-150-3p levels rapidly declined during the early phase of AMI, and we showed that medications may contribute to its early reduction. However, we did not identify a specific medication that was responsible for this effect. A previously study [39] reported a significant downregulation of plasma miR-150 following antiplatelet therapy, suggesting that aspirin and/or clopidogrel might be responsible for the gradual reduction we observed in plasma miR-150-3p levels.

There are also some limitations to the present study. First, future studies including larger populations will be needed to confirm the clinical usefulness of the selected 


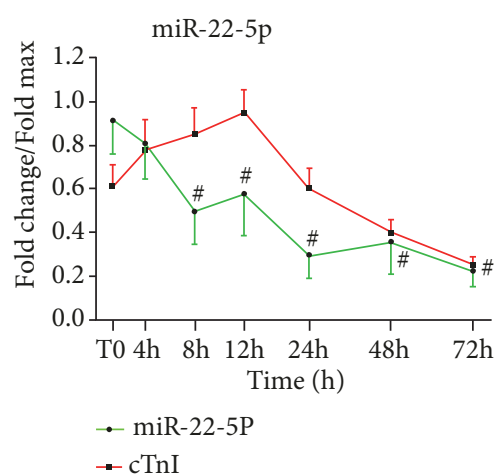

(a)

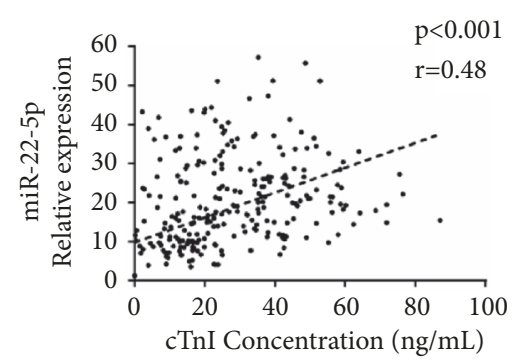

(d)

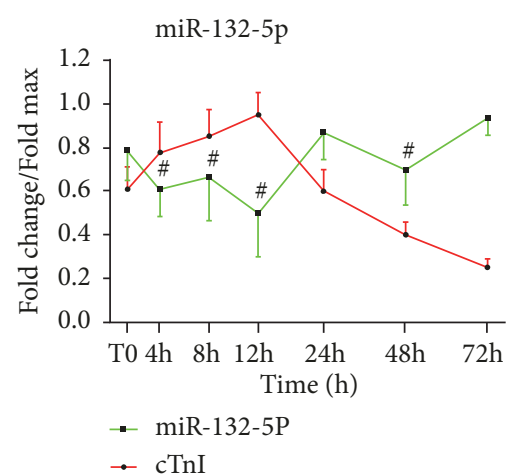

(b)

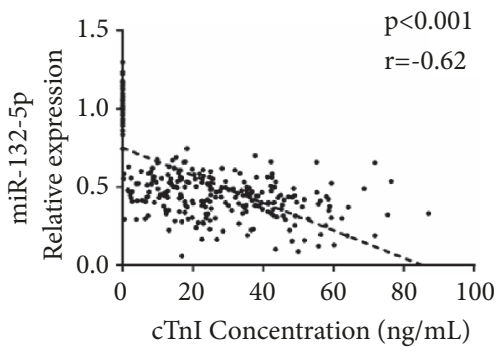

(e)

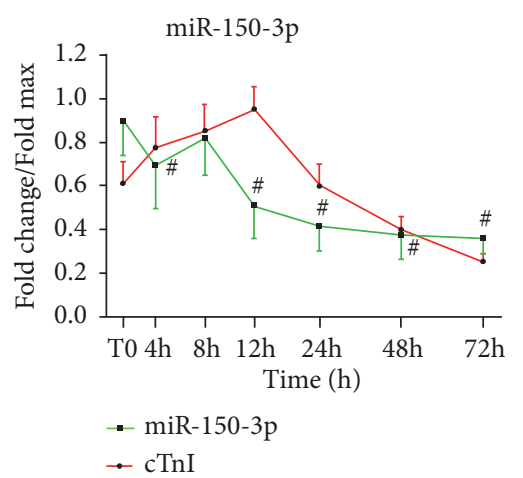

(c)

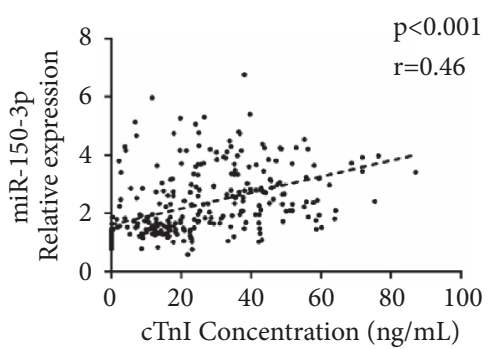

(f)

FIGURE 3: The dynamic expressions of plasma miRNAs (quantitative real-time PCR) and cTnI (immunoassay) in the AMI group and the correlations between them. (a) The expression patterns of plasma miR-22-5p and cTnI in the AMI group; (b) the expression patterns of plasma miR-132-5p and cTnI in the AMI group; and (c) the expression patterns of plasma miR-150-3p and cTnI in the AMI group. (d) The correlation between plasma levels of miR-22-5p and cTnI. (e) The correlation between plasma levels of miR-132-5p and cTnI. (f) The correlation between plasma levels of miR-150-3p and cTnI. Data are shown as the mean \pm SD, \#p $<0.05$ versus peak level in the AMI group. AMI, patients with acute myocardial infarction, $\mathrm{n}=35$; CRTL, control subjects, $\mathrm{n}=55$; cardiac troponin $\mathrm{I}$ (cTnI).

miRNAs in diagnosing AMI. Second, although the expression peaks for miR-22-5p and miR-150-3p occurred earlier than that of cTnI, future studies should determine whether the initial increases in circulating miR-22-5p and miR-150-3p occurs earlier than cTnI. Furthermore, additional studies are required to investigate the mechanisms underlying the dysregulation of these miRNAs and to address whether they are mechanistically involved in the pathophysiological processes underlying AMI.

In conclusion, we have performed the first investigation to examine the dynamic expressions of circulating miR-22$5 p$, miR-132-5p, and miR-150-3p during the early phase of AMI. Our findings indicate that plasma miR-22-5p, miR-132$5 \mathrm{p}$, and miR-150-3p may be considered novel and validated biomarkers for AMI diagnosis. Moreover, a combined panel consisting of these three miRNAs may provide greater diagnostic value for the early identification of AMI.

\section{Materials and Methods}

4.1. Study Design and Ethical Statement. The study protocol was conducted according to the principles expressed in the Declaration of Helsinki and approved by the Medical Ethics Committee of the First Affiliated Hospital of Hebei North University. All participants were inpatients admitted to the Department of Cardiology of First Affiliated Hospital of Hebei North University between December 2015 and August
2017. Written informed consent was obtained from all the involved participants.

In the first cohort, we enrolled 35 consecutive AMI patients and 55 control subjects (CTRL1 group) who presented with chest pain without CHD. The inclusion criteria for AMI patients were based on the most recently developed universal definition of myocardial infarction (MI) [40]. All AMI patients were diagnosed with AMI for the first time and were successfully treated using PCI. A coronary angiography (CAG) or coronary computer tomography angiography (CTA) was performed on each of the control subjects to exclude CHD. Clinical histories and medication records were collected from all participants. The exclusion criteria for all patients were the following: a previous history of impaired left ventricular ejection fraction of $\leq 45 \%$, congestive heart failure, known malignancy, chronic kidney or hepatic diseases, and surgery or skeletal muscle damage within the previous months that would affect the expression of miRNAs.

In the second cohort, we enrolled 40 patients with a history of hypertension and hyperlipidemia who had been on medications for CHD (e.g., ACEI, beta-blockers, nitrates, statins, aspirin, or clopidogrel) for at least 2 weeks into the medication group, and we enrolled 50 age- and gendermatched patients with paroxysmal supraventricular tachycardia (PSVT) who had not taken any of these medications in the past two weeks into the control group (CTRL2 group). 


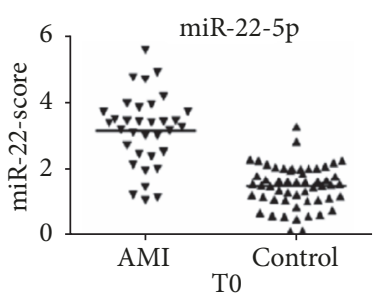

(a)

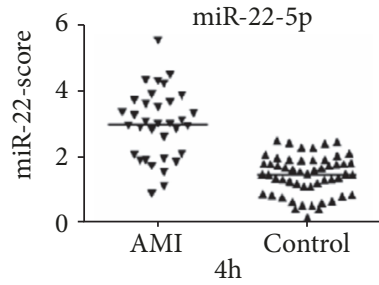

(b)

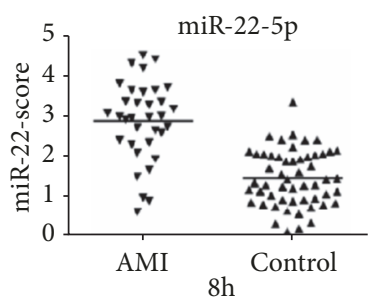

(c)

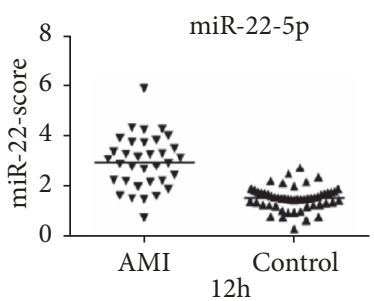

(d)

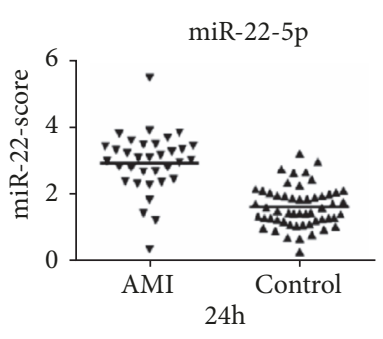

(e)

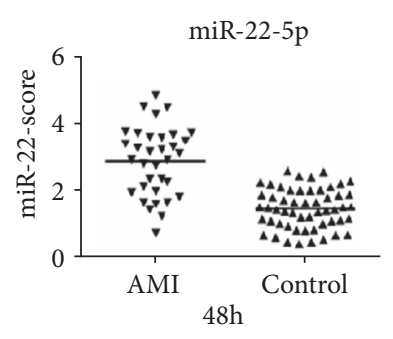

(f)

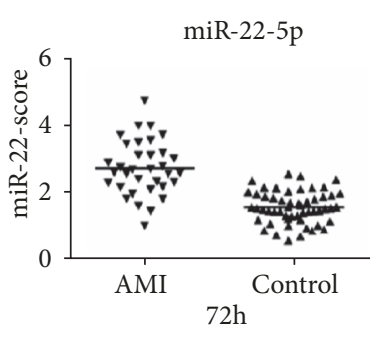

(g)

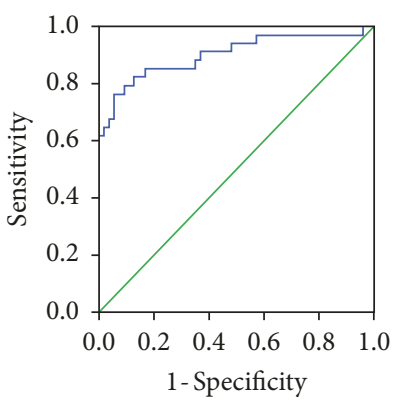

(h)

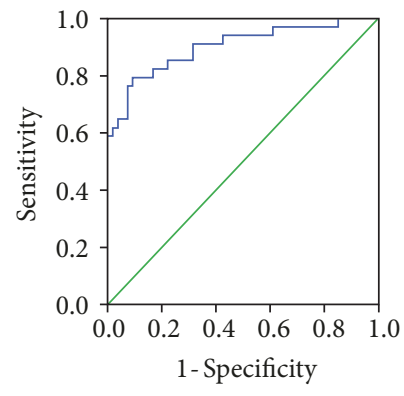

(i)

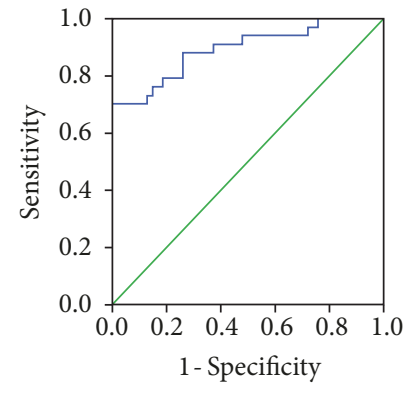

(j)

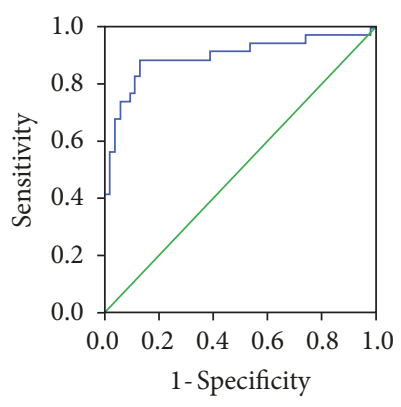

(k)

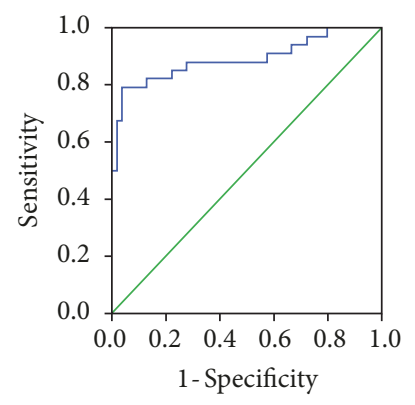

(1)

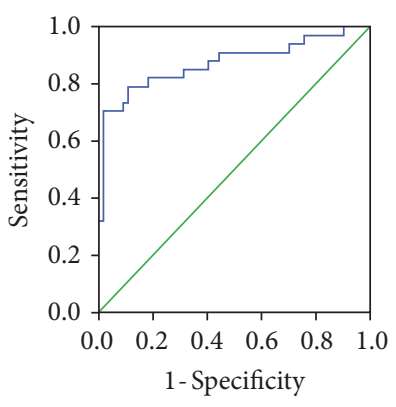

(m)

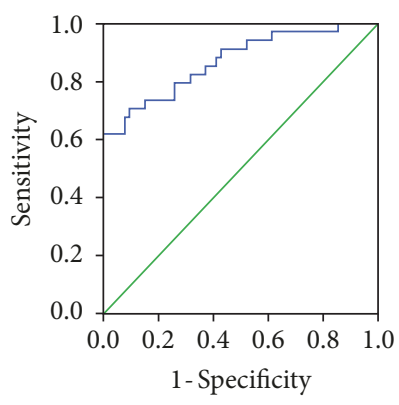

(n)

FIGURE 4: Discriminatory power of plasma miR-22-5p scores. The miR-22-5p scores are presented as the mean values in the AMI and control groups at T0 (a), $4 \mathrm{~h} \mathrm{(b),} 8 \mathrm{~h}$ (c), $12 \mathrm{~h}$ (d), $24 \mathrm{~h}$ (e), $48 \mathrm{~h}$ (f), and $72 \mathrm{~h}$ (g). Receiver operator characteristic (ROC) curves for miR-22-5p at T0 (h), $4 \mathrm{~h}(\mathrm{i}), 8 \mathrm{~h}(\mathrm{j}), 12 \mathrm{~h}(\mathrm{k}), 24 \mathrm{~h}(\mathrm{l}), 48 \mathrm{~h}(\mathrm{~m})$, and $72 \mathrm{~h}(\mathrm{n})$. AMI, patients with acute myocardial infarction, $\mathrm{n}=35$; CRTL, control subjects, $\mathrm{n}=55$.

We then investigated whether these medications affected plasma miRNA expression. The exclusion criteria for the second cohort were the same as those used for the first cohort.

4.2. Blood Sample Collection and Storage. All peripheral venous blood samples were obtained from the AMI patients upon admission to Union hospital. The initial blood sample collection time (T0) was $9.24 \pm 2.81 \mathrm{~h}$ after the onset of AMI symptoms. Subsequent blood samples were obtained at $4 \mathrm{~h} \pm 30 \mathrm{~min}(4 \mathrm{~h}), 12 \mathrm{~h} \pm 30 \mathrm{~min}(12 \mathrm{~h}), 24 \mathrm{~h} \pm 30 \mathrm{~min}$ $(24 \mathrm{~h}), 48 \mathrm{~h} \pm 30 \mathrm{~min}(48 \mathrm{~h})$, and $72 \mathrm{~h} \pm 30 \mathrm{~min}(72 \mathrm{~h})$ after T0. Additionally, plasma was collected pre- and post-PCI procedures to investigate whether heparin had an impact on the expression of selected miRNAs in plasma.

All samples (4-8 mL) were collected into K2-EDTAcoated tubes (BD, NJ, USA). The samples were stored for up to $24 \mathrm{~h}$ at $4^{\circ} \mathrm{C}$ and prepared according to the following 2 step centrifugation protocol: $1,500 \mathrm{x}$ for $15 \mathrm{~min}$ at $4^{\circ} \mathrm{C}$, then $14,000 \mathrm{xg}$ at $4^{\circ} \mathrm{C}$ for another $15 \mathrm{~min}$. After centrifugation, the 


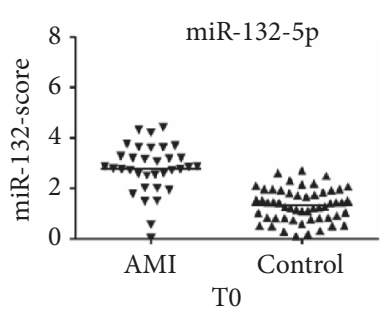

(a)

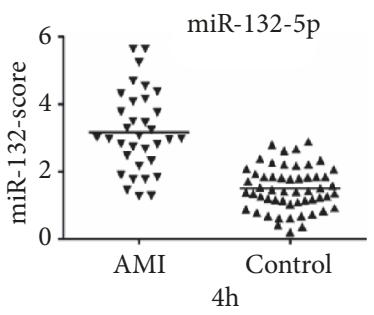

(b)

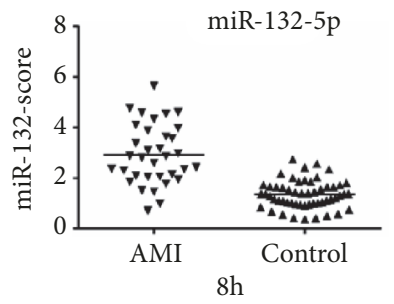

(c)

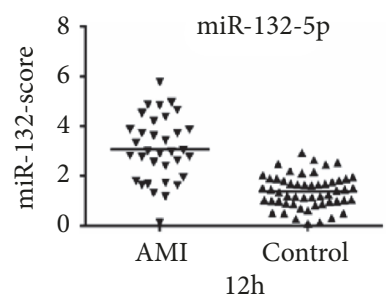

(d)

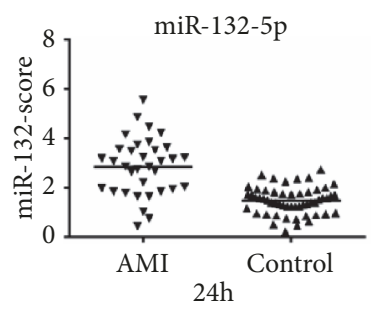

(e)

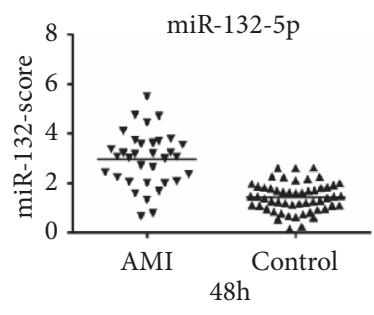

(f)

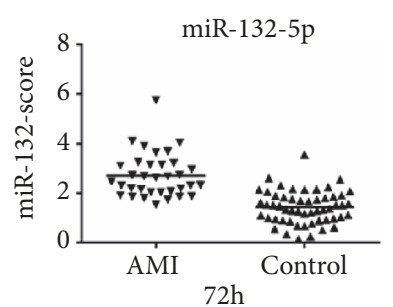

(g)

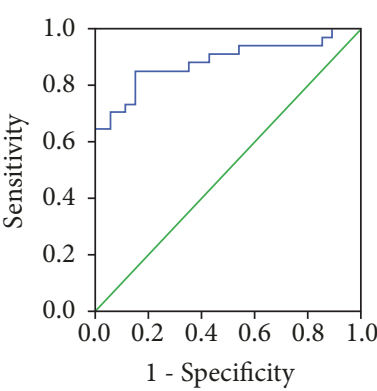

(h)

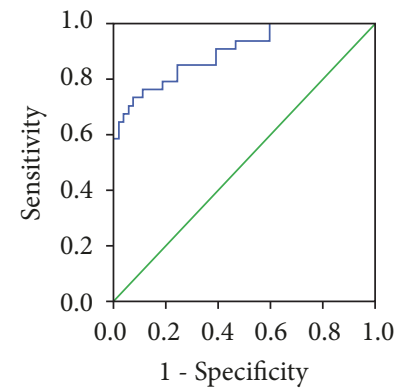

(i)

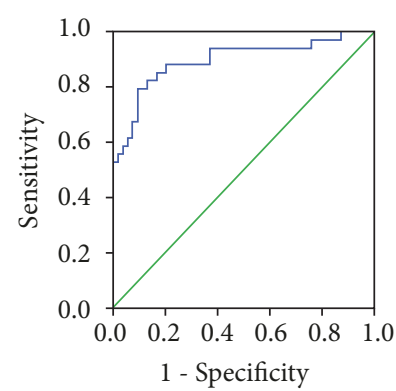

(j)

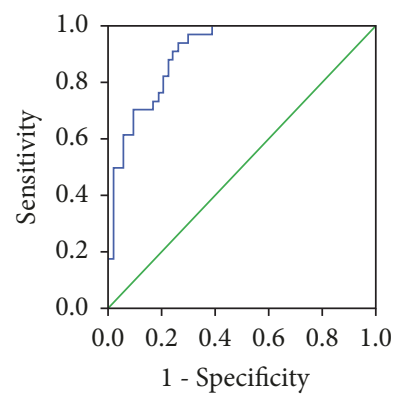

(k)

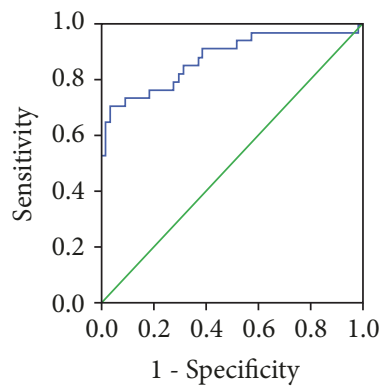

(l)

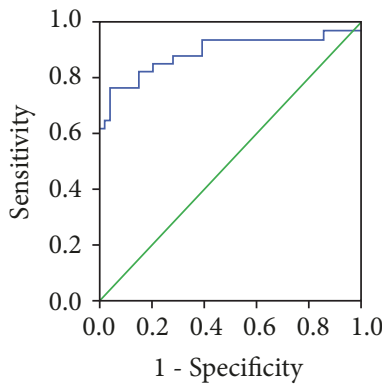

(m)

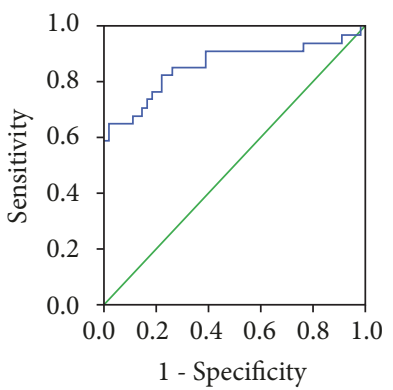

(n)

FIGURE 5: Discriminatory power of plasma miR-132-5p scores. The miR-132-5p scores are presented as the mean values for the AMI and control groups at T0 (a), $4 \mathrm{~h}$ (b), $8 \mathrm{~h}$ (c), $12 \mathrm{~h} \mathrm{(d),24h} \mathrm{(e),} 48 \mathrm{~h}$ (f), and $72 \mathrm{~h}$ (g). Receiver operator characteristic (ROC) curves for miR-132-5p

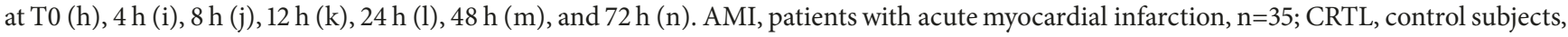
$\mathrm{n}=55$.

supernatant (plasma) was aliquoted into RNase/DNase-free tubes and stored at $-80^{\circ} \mathrm{C}$ for subsequent experiments.

4.3. Isolation of Circulating RNA from Plasma. Total RNA was extracted from frozen plasma using TRIzol Reagent BD (TR126, MRC, Cincinnati, OH, USA) according to the manufacturer's instructions. Briefly, $250 \mu \mathrm{L}$ of plasma was mixed with $750 \mu \mathrm{L}$ of TRIzol (1:3, v/v) and incubated for $15 \mathrm{~min}$ at room temperature to ensure the complete dissociation of nucleoprotein complexes. After $200 \mu \mathrm{L}$ of chloroform was added, the mixture was vortexed vigorously for $15 \mathrm{sec}$. After extraction was performed for $15 \mathrm{~min}$ at room temperature, the mixture was centrifuged at $12,000 \mathrm{x} \mathrm{g}$ for $15 \mathrm{~min}$ at $4^{\circ} \mathrm{C}$. The upper aqueous phase was transferred to fresh reagent tubes, an equal volume of cold isopropanol was added, and the mixture was stored at $-20^{\circ} \mathrm{C}$ overnight. The samples were centrifuged at $12,000 \mathrm{x}$ g for $15 \mathrm{~min}$ at $4^{\circ} \mathrm{C}$. The supernatant was then removed, and the RNA pellet was washed with $1 \mathrm{~mL}$ of $75 \%(\mathrm{v} / \mathrm{v})$ ethanol to remove residual salt. For normalization, each sample was supplemented with $25 \mathrm{pM}$ Caenorhabditis elegans miR-39 (cel-miR-39) after the TRIzol was added, as previously reported [41]. 


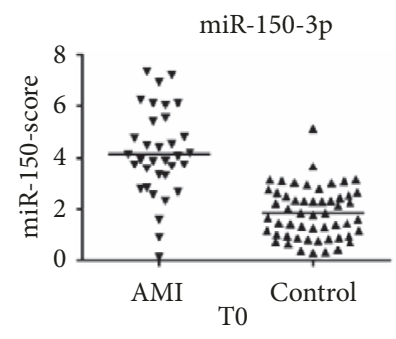

(a)

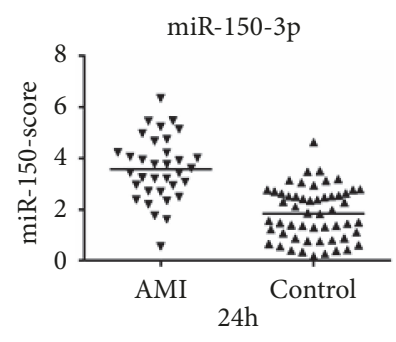

(e)

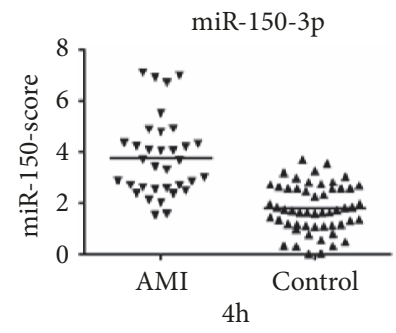

(b)

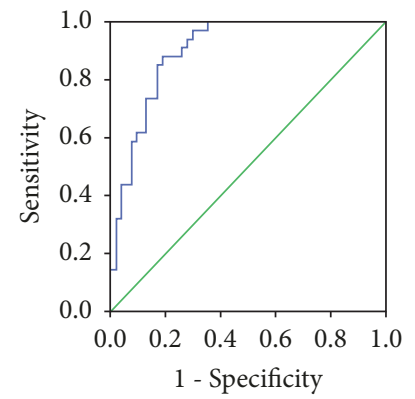

(f)

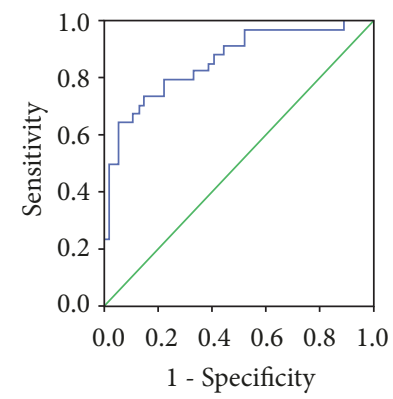

(i)

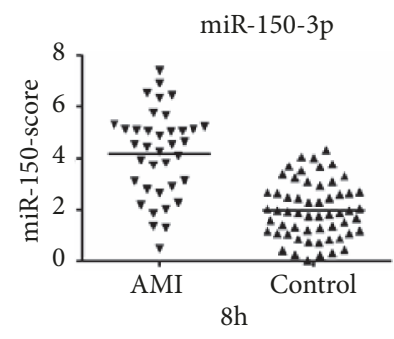

(c)

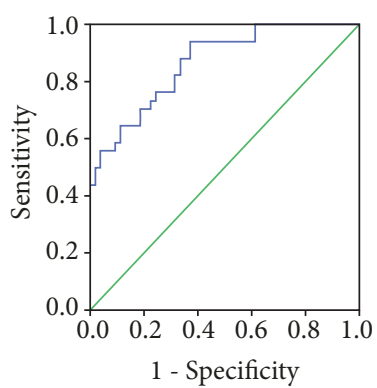

(g)

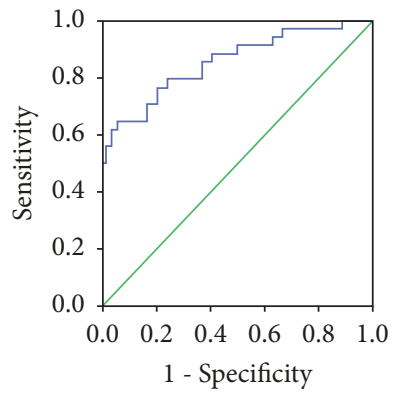

(j)

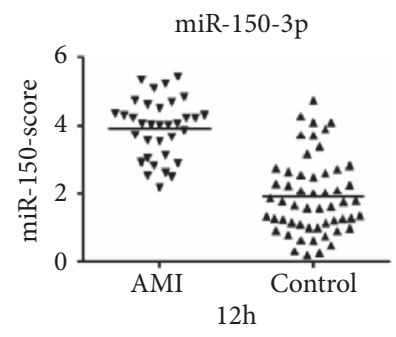

(d)

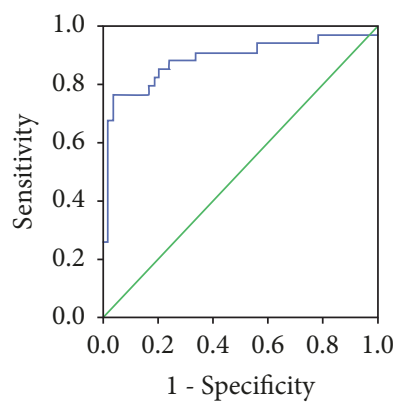

(h)

FIGURE 6: Discriminatory power of plasma miR-150-3p scores. The miR-150-3p scores are presented as the mean values for the AMI and

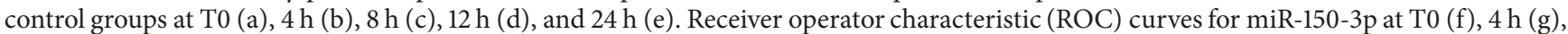
$8 \mathrm{~h}(\mathrm{~h}), 12 \mathrm{~h}(\mathrm{i})$, and $24 \mathrm{~h}(\mathrm{j})$. AMI, patients with acute myocardial infarction, $\mathrm{n}=35$; CRTL, control subjects, $\mathrm{n}=55$.

\section{MicroRNA Analysis}

Total RNA $(1 \mu \mathrm{g})$ was reverse-transcribed using a cDNA Reverse Transcription Kit (TAKARA, Shiga, Japan) according to the manufacturer's protocol. Briefly, the mixed reagents were incubated for $60 \mathrm{~min}$ at $42^{\circ} \mathrm{C}, 10 \mathrm{~min}$ at $70^{\circ} \mathrm{C}$, and then $4^{\circ} \mathrm{C}$ for $5 \mathrm{~min}$. qRT-PCR was performed using cel-miR-39 as the normalization control with a Bulge-Loop ${ }^{\mathrm{TM}}$ miRNA RT-qPCR Detection Kit (Ribobio, Guangzhou, China) and SYBR Green PCR Master Mix Kit (TAKARA, Shiga, Japan). Briefly, the reactions were incubated at $95^{\circ} \mathrm{C}$ for $20 \mathrm{~s}$, followed by 40 cycles of $95^{\circ} \mathrm{C}$ for $10 \mathrm{~s}, 60^{\circ} \mathrm{C}$ for $20 \mathrm{~s}$, and $70^{\circ} \mathrm{C}$ for $1 \mathrm{~s}$ (a cycle threshold $(\mathrm{Ct}) \geq 40$ was considered to indicate undetermined). All data were analyzed using BioRad CFX Manager software (Bio-Rad, CA, USA). The Ct values were normalized to cel-miR-39 using the formula $2^{-(\mathrm{Ct}[\mathrm{miRNA}]-\mathrm{Ct}[\mathrm{cel}-\mathrm{miRNA}-39])}$, and the $2^{-\Delta \Delta \mathrm{Ct}}$ method was used to analyze the relative expression levels of the miRNAs.

5.1. Cardiac Troponin I Analysis. All plasma samples were stored at $-80^{\circ} \mathrm{C}$ prior to analysis. All frozen plasma samples were thawed and deidentified for research and clinical purposes. Then, using an sandwich chemiluminescent magnetic microparticle immunoassay method, the Abbott-Architect Troponin I assay (Abbott Diagnostics, Abbott Park, IL, USA) was performed, with a limit of detection of $0.01 \mathrm{ng} / \mathrm{ml}$, a 99thpercentile cutoff point of $0.028 \mathrm{ng} / \mathrm{ml}$, and a coefficient of variation $(\mathrm{CV})$ of less than $10 \%$ at $0.032 \mathrm{ng} / \mathrm{ml}$, as specified by the manufacturer. The normal reference range for $\mathrm{cTnI}$ was established as $<0.03 \mathrm{ng} / \mathrm{ml}$.

5.2. Statistical Methods. All data were presented as the mean \pm standard deviation (SD). Student's two-sided $t$ tests or one-way ANOVA followed by Bonferroni's multiple comparison tests (as a post hoc analysis) were used for normally distributed values. For variables without a normal distribution, the Kruskal-Wallis and Mann-Whitney tests were performed. The time-courses of the expression levels of the miRNAs and cTnI were analyzed using repeatedmeasures ANOVA. For categorical clinical variables, the ChiSquare $(\chi 2)$ test (or Fisher's exact test when necessary) was used. The correlation analysis of miRNAs and cTnI was 


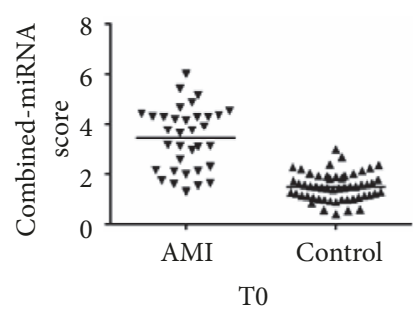

(a)

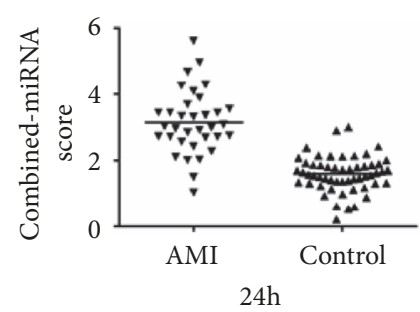

(e)

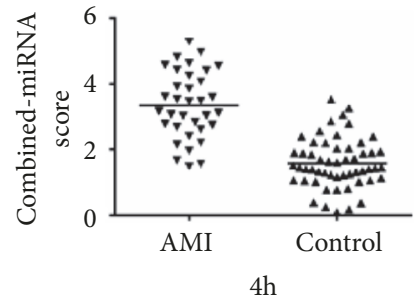

(b)

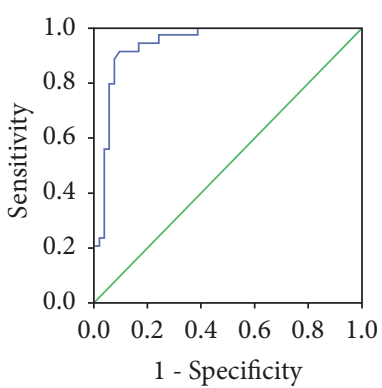

(f)

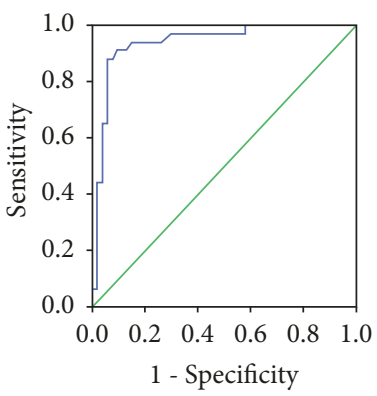

(i)

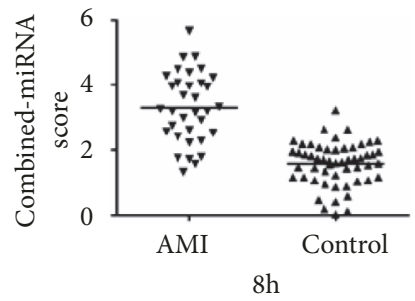

(c)

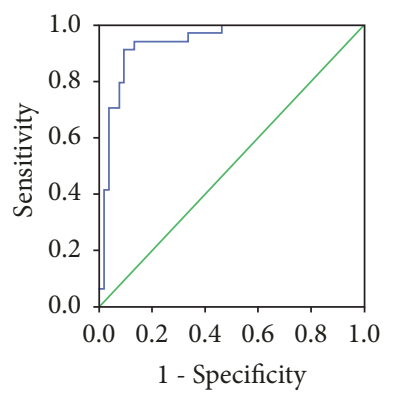

(g)

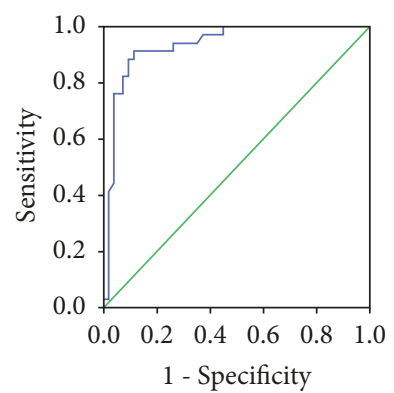

(j)

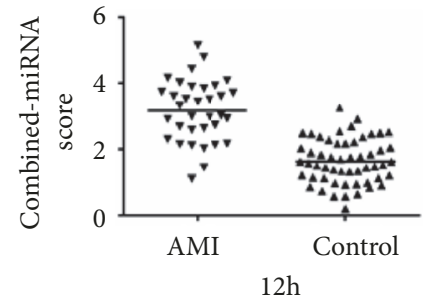

(d)

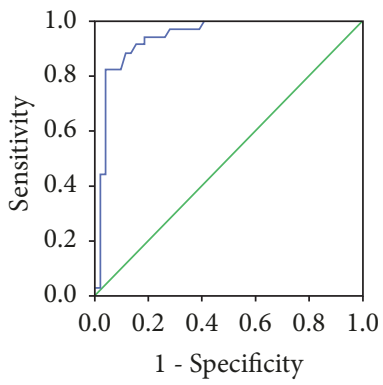

(h)

FIGURE 7: Discriminatory power of combined-miRNA scores. The combined-miRNA scores are presented as the mean values for the AMI and control groups at T0 (a), $4 \mathrm{~h} \mathrm{(b),} 8 \mathrm{~h}$ (c), $12 \mathrm{~h}$ (d), and $24 \mathrm{~h}$ (e). Receiver operator characteristic (ROC) curves for miR-150-3p at T0 (f), $4 \mathrm{~h}$ $(\mathrm{g}), 8 \mathrm{~h}(\mathrm{~h}), 12 \mathrm{~h}(\mathrm{i})$, and $24 \mathrm{~h}(\mathrm{j})$. The combined-miRNA scores include the scores for miR-22-5p, miR-132-5p, and miR-150-3p. AMI, patients with acute myocardial infarction, $\mathrm{n}=35$; CRTL, control subjects, $\mathrm{n}=55$.

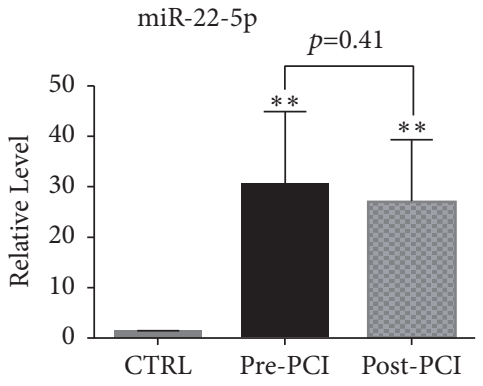

(a)
miR-132-5p

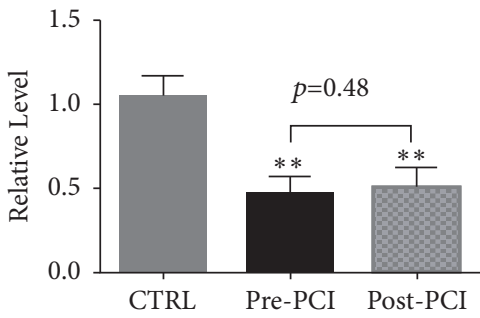

(b)
miR-150-3p

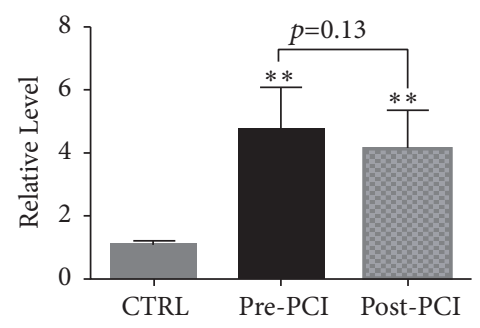

(c)

FIGURE 8: The impact of heparin on plasma miRNA (Quantitative real-time PCR) expression levels during the PCI procedure. The expression levels of circulating miR-22-5p (a), miR-132-5p (b), and miR-150-3p (c) in the pre-PCI and post-PCI plasma of AMI patients. AMI, patients with acute myocardial infarction; PCI, percutaneous coronary intervention; CRTL, control subjects. Data are shown as the mean \pm SD, $* * \mathrm{p}<0.01$ versus CTRL. Pre-PCI, previous-percutaneous coronary intervention, $\mathrm{n}=35$; Post-PCI, postprocedure percutaneous coronary intervention, $\mathrm{n}=35$; CRTL, control subjects, $\mathrm{n}=55$. 
TABLE 4: Diagnostic value of circulating selected-miRNAs for AMI.

\begin{tabular}{|c|c|c|c|c|c|c|c|}
\hline Time point & miRNAs & AUC & $95 \% \mathrm{CI}$ & $\mathrm{p}$ value & Cut off value & Sensitivity & Specificity \\
\hline \multirow[t]{4}{*}{ T0 } & miR-22-5p & 0.901 & $0.827-0.976$ & $\mathrm{p}<0.001$ & 1.9837 & 0.882 & 0.759 \\
\hline & miR-132-5p & 0.886 & $0.805-0.967$ & $\mathrm{p}<0.001$ & 1.8314 & 0.853 & 0.741 \\
\hline & miR-150-3p & 0.904 & $0.843-0.964$ & $\mathrm{p}<0.001$ & 2.5457 & 0.882 & 0.759 \\
\hline & Combined score & 0.942 & $0.893-0.991$ & $\mathrm{p}<0.001$ & 2.2850 & 0.912 & 0.870 \\
\hline \multirow[t]{4}{*}{$4 \mathrm{~h}$} & miR-22-5p & 0.901 & $0.831-0.972$ & $\mathrm{p}<0.001$ & 1.8764 & 0.853 & 0.741 \\
\hline & miR-132-5p & 0.900 & $0.833-0.967$ & $\mathrm{p}<0.001$ & 1.8517 & 0.853 & 0.741 \\
\hline & miR-150-3p & 0.867 & $0.793-0.941$ & $\mathrm{p}<0.001$ & 2.5124 & 0.794 & 0.685 \\
\hline & Combined score & 0.938 & $0.886-0.990$ & $\mathrm{p}<0.001$ & 2.2085 & 0.941 & 0.842 \\
\hline \multirow[t]{4}{*}{$8 \mathrm{~h}$} & miR-22-5p & 0.895 & $0.822-0.968$ & $\mathrm{p}<0.001$ & 1.8569 & 0.853 & 0.741 \\
\hline & miR-132-5p & 0.897 & $0.823-0.967$ & $\mathrm{p}<0.001$ & 1.6987 & 0.882 & 0.759 \\
\hline & miR-150-3p & 0.887 & $0.805-0.970$ & $\mathrm{p}<0.001$ & 2.4592 & 0.882 & 0.759 \\
\hline & Combined score & 0.943 & 0.894-0.992 & $\mathrm{p}<0.001$ & 2.1300 & 0.912 & 0.815 \\
\hline \multirow[t]{4}{*}{$12 \mathrm{~h}$} & miR-22-5p & 0.895 & $0.815-0.975$ & $\mathrm{p}<0.001$ & 2.0128 & 0.882 & 0.741 \\
\hline & miR-132-5p & 0.909 & $0.851-0.967$ & $\mathrm{p}<0.001$ & 1.7088 & 0.912 & 0.759 \\
\hline & miR-150-3p & 0.859 & $0.778-0.941$ & $\mathrm{p}<0.001$ & 2.6464 & 0.794 & 0.704 \\
\hline & Combined score & 0.941 & $0.888-0.994$ & $\mathrm{p}<0.001$ & 2.0900 & 0.912 & 0.852 \\
\hline \multirow[t]{4}{*}{$24 \mathrm{~h}$} & miR-22-5p & 0.893 & $0.814-0.972$ & $\mathrm{p}<0.001$ & 1.9837 & 0.853 & 0.741 \\
\hline & miR-132-5p & 0.878 & $0.798-0.958$ & $\mathrm{p}<0.001$ & 1.7683 & 0.824 & 0.704 \\
\hline & miR-150-3p & 0.853 & $0.768-0.939$ & $\mathrm{p}<0.001$ & 2.5121 & 0.794 & 0.704 \\
\hline & Combined score & 0.936 & 0.883-0.989 & $\mathrm{p}<0.001$ & 2.1700 & 0.912 & 0.796 \\
\hline \multirow[t]{2}{*}{$48 \mathrm{~h}$} & miR-22-5p & 0.874 & $0.789-0.959$ & $\mathrm{P}=0.001$ & 1.9946 & 0.824 & 0.722 \\
\hline & miR-132-5p & 0.895 & $0.814-0.976$ & $\mathrm{p}<0.001$ & 1.8083 & 0.853 & 0.741 \\
\hline \multirow[t]{2}{*}{$72 \mathrm{~h}$} & miR-22-5p & 0.870 & $0.791-0.950$ & $\mathrm{P}=0.001$ & 1.8744 & 0.794 & 0.704 \\
\hline & miR-132-5p & 0.859 & $0.768-0.951$ & $\mathrm{p}<0.001$ & 1.8334 & 0.853 & 0.722 \\
\hline
\end{tabular}

Combined score, the combination of miR-22-5p, miR-132-5p, and miR-150-3p scores; AUC, area under the ROC curve; 95\% Cl, 95\% confidence interval.

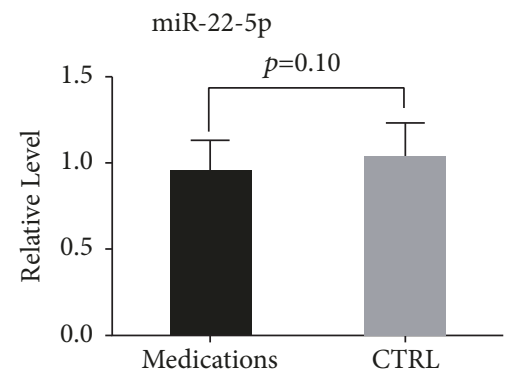

(a)

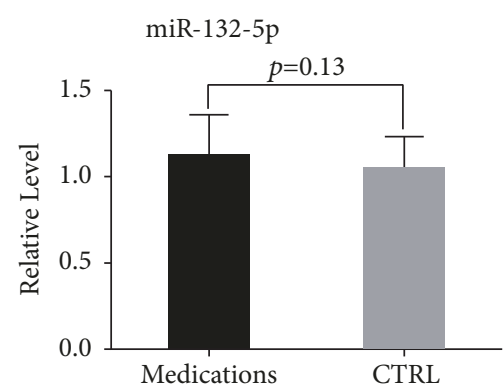

(b)

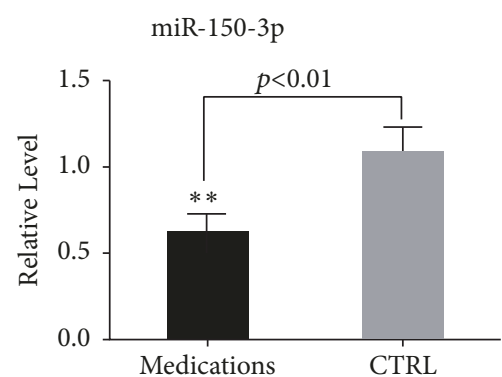

(c)

FiguRE 9: The impact of CHD medications on plasma miRNA expression levels. The expression of plasma miR-22-5p (a), miR-132-5p (b), and miR-150-3p (c) in the medication and control groups. Medications, patients taking medications, $n=40$; CRTL, patients without medications, $\mathrm{n}=50$. Data are shown as the mean $\pm \mathrm{SD} ; * * p<0.01$ versus CTRL. Coronary heart disease CHD.

performed using a linear regression analysis. All statistical tests were two-tailed, and $\mathrm{p}<0.05$ was considered statistically significant.

The ROC curves for the plasma miRNAs were analyzed to discriminate AMI patients from control subjects, and the areas under the ROC curves (AUCs) were estimated to assess the diagnostic accuracy of the identified miRNAs. Herein, an inverted-normalized miRNA signal (the miRNA-score) was used to represent the relative expression level of the selected miRNAs in the AMI group in comparison to the control group. As previously reported [17, 18], the miRNA scores were calculated by deducting the normalized $\mathrm{Ct}$ from 40 and then adjusted by subtracting the minimal score (so that the miRNA scores started at 0 ). The optimal diagnostic points for each of the miRNAs were assessed at cutoff values using the largest Youden's index (sensitivity + specificity - 1). All statistical analyses were performed using SPSS 13.0 (Chicago, IL, USA).

\section{Abbreviations}

ACEI: Angiotensin converting enzyme inhibitor

AMI: Acute myocardial infarction 


$\begin{array}{ll}\text { AUC: } & \text { Area under the ROC curve } \\ \text { BMI: } & \text { Body mass index } \\ \text { CAG: } & \text { Coronary angiography } \\ \text { CHD: } & \text { Coronary heart disease } \\ \text { CI: } & \text { Confidence interval } \\ \text { CK-MB: } & \text { Creatine kinase-MB } \\ \text { Cr: } & \text { Creatinine } \\ \text { cTnI/T: } & \text { Cardiac troponin I/T } \\ \text { DBP: } & \text { Diastolic blood pressure } \\ \text { HDL: } & \text { High-density lipoprotein } \\ \text { LDL: } & \text { Low-density lipoprotein } \\ \text { miRNAs or miRs: } & \text { MicroRNAs } \\ \text { mRNAs: } & \text { Messenger RNAs } \\ \text { PCI: } & \text { Percutaneous coronary intervention } \\ \text { PSVT: } & \text { Paroxysmal supraventricular tachycardia } \\ \text { qRT-PCR: } & \text { Quantitative real-time PCR } \\ \text { ROC: } & \text { Receiver operating characteristic } \\ \text { SBP: } & \text { Systolic blood pressure } \\ \text { SD: } & \text { Standard deviation } \\ \text { TC: } & \text { Total cholesterol } \\ \text { TG: } & \text { Total triglyceride } \\ \text { UAP: } & \text { Unstable angina pectoris } \\ \text { WBC: } & \text { White blood cell. } \\ & \end{array}$

\section{Data Availability}

The data used to support the findings of this study are available from the corresponding author upon request.

\section{Conflicts of Interest}

The authors declare that they have no conflicts of interest.

\section{Authors' Contributions}

Huixian Li and Pengxiang Zhang contributed equally to this work.

\section{Acknowledgments}

This work was supported by a grant from the Science and Technology Department of Hebei Province (17277792D).

\section{References}

[1] A. D. Lopez, C. D. Mathers, M. Ezzati, D. T. Jamison, and C. J. Murray, "Global and regional burden of disease and risk factors, 2001: systematic analysis of population health data," The Lancet, vol. 367, no. 9524, pp. 1747-1757, 2006.

[2] H. D. White and D. P. Chew, "Acute myocardial infarction," The Lancet, vol. 372, no. 9638, pp. 570-584, 2008.

[3] C. Chen, J. Xu, and F. Huang, "Recent players in the field of acute myocardial infarction biomarkers: Circulating cell-free DNA or microRNAs?" International Journal of Cardiology, vol. 168, no. 3, pp. 2956-2957, 2013.

[4] V. Ambros, "The functions of animal microRNAs," Nature, vol. 431, no. 7006, pp. 350-355, 2004.
[5] Y. Lee, C. Ahn, J. Han et al., “The nuclear RNase III Drosha initiates microRNA processing," Nature, vol. 425, no. 6956, pp. 415-419, 2003.

[6] J. G. Doench and P. A. Sharp, "Specificity of microRNA target selection in translational repression," Genes \& Development, vol. 18, no. 5, pp. 504-511, 2004.

[7] D. P. Bartel, "MicroRNAs: genomics, biogenesis, mechanism, and function," Cell, vol. 116, no. 2, pp. 281-297, 2004.

[8] M. A. Cortez, C. Bueso-Ramos, J. Ferdin, G. Lopez-Berestein, A. K. Sood, and G. A. Calin, "MicroRNAs in body fluids-the mix of hormones and biomarkers," Nature Reviews Clinical Oncology, vol. 8, no. 8, pp. 467-477, 2011.

[9] C. Cheng, Q. Wang, W. You, M. Chen, and J. Xia, "MiRNAs as biomarkers of myocardial infarction: a meta-analysis," PLoS ONE, vol. 9, no. 2, Article ID e88566, 2014.

[10] Y. D’Alessandra, P. Devanna, F. Limana et al., "Circulating microRNAs are new and sensitive biomarkers of myocardial infarction," European Heart Journal, vol. 31, no. 22, pp. 27652773, 2010

[11] Z.-P. Huang, J. Chen, H. Y. Seok et al., "MicroRNA-22 regulates cardiac hypertrophy and remodeling in response to stress," Circulation Research, vol. 112, no. 9, pp. 1234-1243, 2013.

[12] A. Ucar, S. K. Gupta, J. Fiedler et al., "The miRNA-212/132 family regulates both cardiac hypertrophy and cardiomyocyte autophagy," Nature Communications, vol. 3, article 1078, 2012.

[13] Y. Tang, Y. Wang, K. M. Park et al., "MicroRNA-150 protects the mouse heart from ischaemic injury by regulating cell death," Cardiovascular Research, vol. 106, no. 3, pp. 387-397, 2015.

[14] T. Huan, J. Rong, K. Tanriverdi et al., "Dissecting the roles of MicroRNAs in coronary heart disease via integrative genomic analyses," Arteriosclerosis, Thrombosis, and Vascular Biology, vol. 35, no. 4, pp. 1011-1021, 2015.

[15] W. Liu, Y. Liu, Y. Zhang et al., "MicroRNA-150 protects against pressure overload-induced cardiac hypertrophy," Journal of Cellular Biochemistry, vol. 116, no. 10, pp. 2166-2176, 2015.

[16] Y. Duan, B. Zhou, H. Su, Y. Liu, and C. Du, "MiR-150 regulates high glucose-induced cardiomyocyte hypertrophy by targeting the transcriptional co-activator p300," Experimental Cell Research, vol. 319, no. 3, pp. 173-184, 2013.

[17] Y. Goren, M. Kushnir, B. Zafrir, S. Tabak, B. S. Lewis, and O. Amir, "Serum levels of microRNAs in patients with heart failure," European Journal of Heart Failure, vol. 14, no. 2, pp. 147154, 2012.

[18] K.-J. Wang, X. Zhao, Y.-Z. Liu et al., "Circulating MiR-19b-3p, MiR-134-5p and MiR-186-5p are promising novel biomarkers for early diagnosis of acute myocardial infarction," Cellular Physiology and Biochemistry, vol. 38, no. 3, pp. 1015-1029, 2016.

[19] D. Kaudewitz, R. Lee, P. Willeit et al., "Impact of intravenous heparin on quantification of circulating microRNAs in patients with coronary artery disease," Thrombosis and Haemostasis, vol. 110, no. 3, pp. 609-615, 2013.

[20] T. Thum, D. Catalucci, and J. Bauersachs, "MicroRNAs: novel regulators in cardiac development and disease," Cardiovascular Research, vol. 79, no. 4, pp. 562-570, 2008.

[21] J. M. Lorenzen and T. Thum, "Long noncoding RNAs in kidney and cardiovascular diseases," Nature Reviews Nephrology, vol. 12, no. 6, pp. 360-373, 2016.

[22] M. W. Feinberg and K. J. Moore, "MicroRNA regulation of atherosclerosis," Circulation Research, vol. 118, no. 4, pp. 703720, 2016. 
[23] Q. Yang, C. Jia, and P. Wang, "MicroRNA-505 identified from patients with essential hypertension impairs endothelial cell migration and tube formation," International Journal of Cardiology, vol. 177, no. 3, pp. 925-934, 2014.

[24] S. Fichtlscherer, A. M. Zeiher, and S. Dimmeler, "Circulating MicroRNAs: biomarkers or mediators of cardiovascular diseases?" Arteriosclerosis, Thrombosis, and Vascular Biology, vol. 31, no. 11, pp. 2383-2390, 2011.

[25] R. Navickas, D. Gal, A. Laucevičius, A. Taparauskaite, M. Zdanyte, and P. Holvoet, "Identifying circulating microRNAs as biomarkers of cardiovascular disease: a systematic review," Cardiovascular Research, vol. 111, no. 4, pp. 322-337, 2016.

[26] T. Zeller, T. Keller, F. Ojeda et al., "Assessment of microRNAs in patients with unstable angina pectoris," European Heart Journal, vol. 35, no. 31, pp. 2106-2114, 2014.

[27] D. D. McManus, H. Lin, K. Tanriverdi et al., "Relations between circulating microRNAs and atrial fibrillation: data from the Framingham Offspring Study," Heart Rhythm, vol. 11, no. 4, pp. 663-669, 2014.

[28] V. Fuster, P. R. Moreno, Z. A. Fayad, R. Corti, and J. J. Badimon, "Atherothrombosis and high-risk plaque: part I: evolving concepts," Journal of the American College of Cardiology, vol. 46, no. 6, pp. 937-954, 2005.

[29] X.-D. Xu, X.-W. Song, Q. Li, G.-K. Wang, Q. Jing, and Y.-W. Qin, "Attenuation of microRNA-22 derepressed PTEN to effectively protect rat cardiomyocytes from hypertrophy," Journal of Cellular Physiology, vol. 227, no. 4, pp. 1391-1398, 2012.

[30] J. Yang, J. Yang, L. Chen et al., "MicroRNA-22 targeting CBP protects against myocardial ischemia-reperfusion injury through anti-apoptosis in rats," Molecular Biology Reports, vol. 41, no. 1, pp. 555-561, 2014.

[31] S. Coffey, M. J. Williams, L. V. Phillips, and G. T. Jones, "Circulating microRNA profiling needs further refinement before clinical use in patients with aortic stenosis," Journal of the American Heart Association, vol. 4, no. 8, Article ID e002150, 2015.

[32] T. Franchina, V. Amodeo, G. Bronte et al., "Circulating miR22 , miR-24 and miR-34a as novel predictive biomarkers to pemetrexed-based chemotherapy in advanced non-small cell lung cancer," Journal of Cellular Physiology, vol. 229, no. 1, pp. 97-99, 2014.

[33] S. Anand, B. K. Majeti, L. M. Acevedo et al., "MicroRNA-132mediated loss of p120RasGAP activates the endothelium to facilitate pathological angiogenesis," Nature Medicine, vol. 16, no. 8, pp. 909-914, 2010.

[34] Z. Lei, A. van Mil, M. M. Brandt et al., "MicroRNA-132/212 family enhances arteriogenesis after hindlimb ischaemia through modulation of the Ras-MAPK pathway," Journal of Cellular and Molecular Medicine, vol. 19, no. 8, pp. 1994-2005, 2015.

[35] T. V. Eskildsen, P. L. Jeppesen, M. Schneider et al., "Angiotensin II regulates microRNA-132/-212 in hypertensive rats and humans," International Journal of Molecular Sciences, vol. 14, no. 6, pp. 11190-11207, 2013.

[36] Z. Liu, P. Ye, S. Wang et al., "MicroRNA-150 protects the heart from injury by inhibiting monocyte accumulation in a mouse model of acute myocardial infarction," Circulation: Cardiovascular Genetics, vol. 8, no. 1, pp. 11-20, 2015.

[37] C. Roderburg, M. Luedde, D. Vargas Cardenas et al., "Circulating microRNA-150 serum levels predict survival in patients with critical illness and sepsis," PLoS ONE, vol. 8, no. 1, Article ID e54612, 2013.
[38] Y. Devaux, M. Vausort, G. P. McCann et al., "MicroRNA150: A novel marker of left ventricular remodeling after acute myocardial infarction," Circulation: Cardiovascular Genetics, vol. 6, no. 3, pp. 290-298, 2013.

[39] P. Willeit, A. Zampetaki, K. Dudek et al., "Circulating MicroRNAs as novel biomarkers for platelet activation," Circulation Research, vol. 112, no. 4, pp. 595-600, 2013.

[40] K. Thygesen, J. S. Alpert, A. S. Jaffe, M. L. Simoons, B. R. Chaitman, and H. D. White, "Third universal definition of myocardial infarction," Journal of the American College of Cardiology, vol. 60, no. 16, pp. 1581-1598, 2012.

[41] S. Huang, M. Chen, L. Li et al., "Circulating microRNAs and the occurrence of acute myocardial infarction in chinese populations," Circulation: Cardiovascular Genetics, vol. 7, no. 2, pp. 189-198, 2014. 


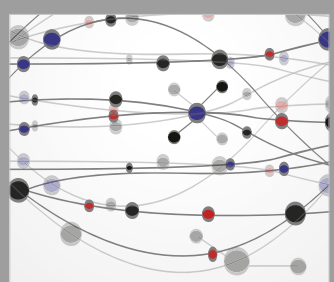

The Scientific World Journal
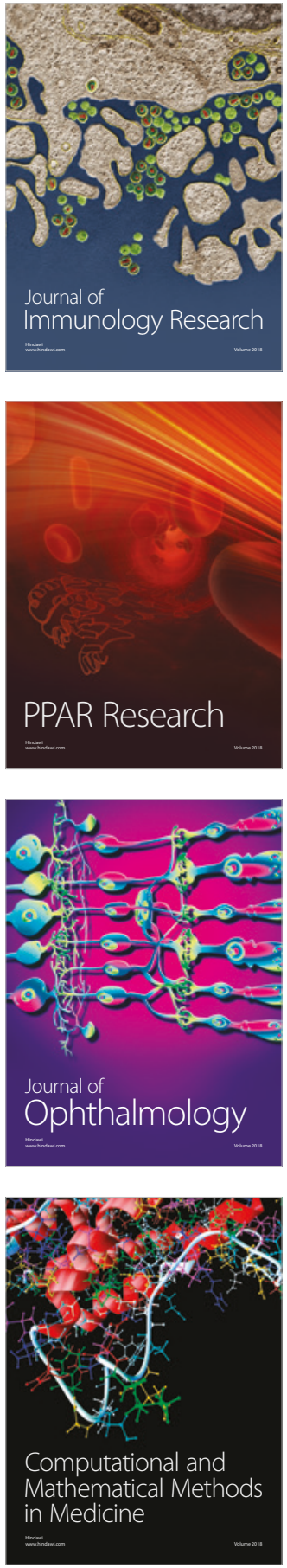

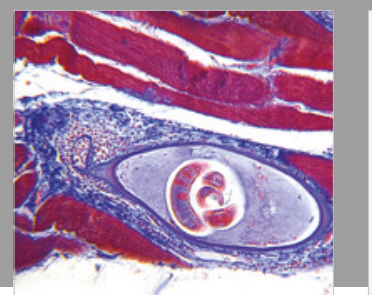

Gastroenterology Research and Practice

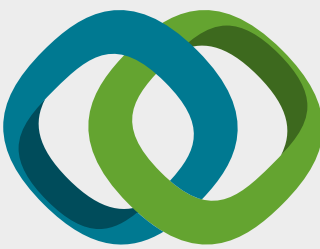

\section{Hindawi}

Submit your manuscripts at

www.hindawi.com
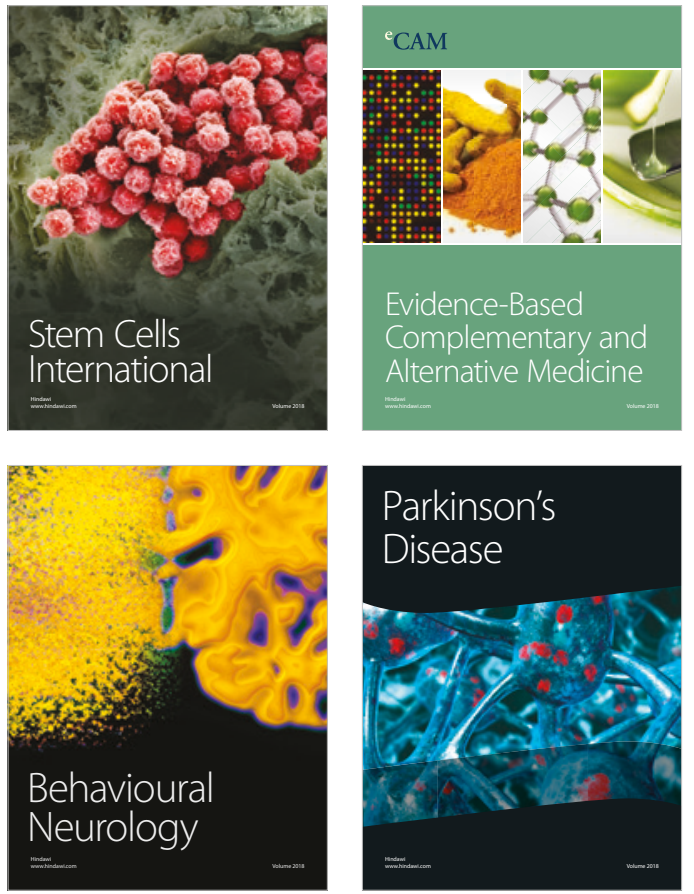

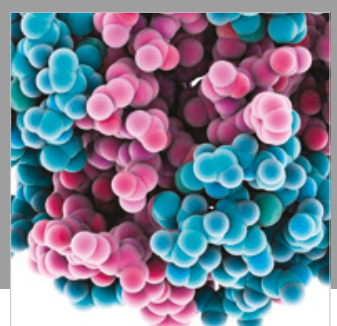

ournal of

Diabetes Research

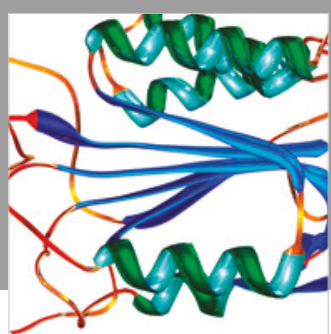

Disease Markers
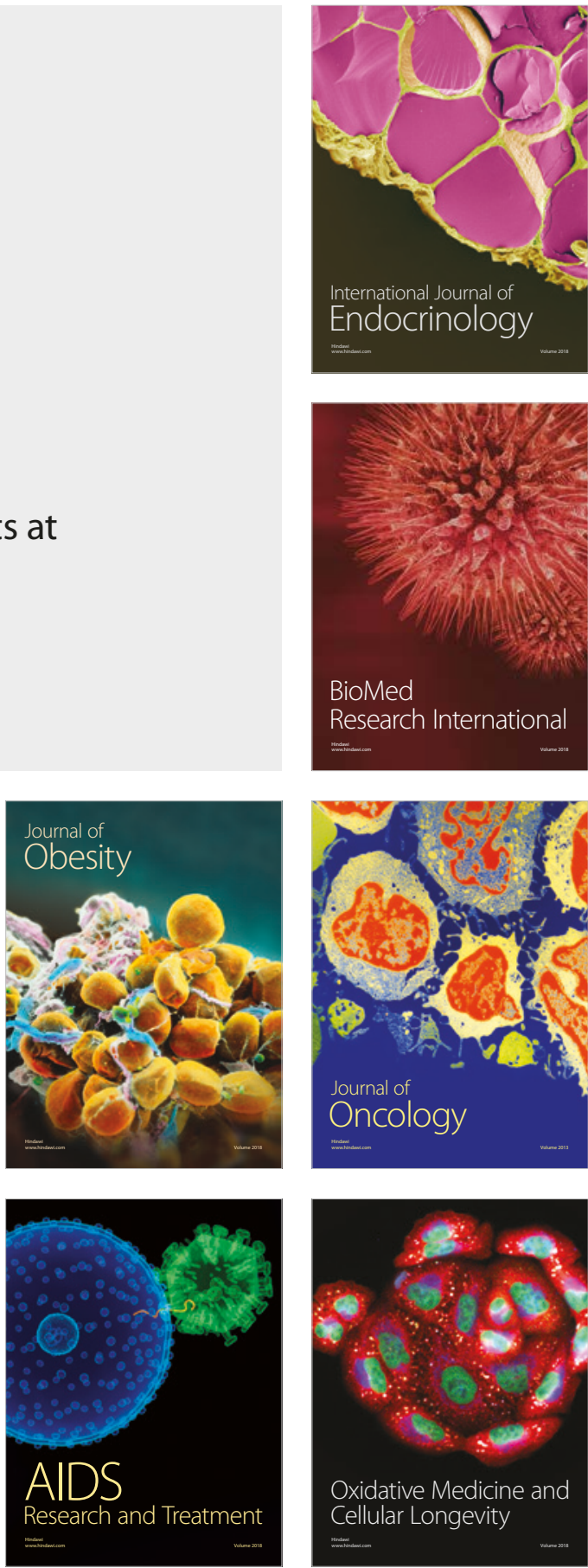